\title{
INCIDENCTA DE ACIDENTE CEREBRO-VASCULAR EMBÓLICO NA CARDIOPATIA CHAGÁSICA CRÔNICA
}

\author{
A. SPINA-FranÇa * \\ N. YASUDA $* *$
}

Andrade ${ }^{3}$, em avaliação crítica dos aspectos anátomo-patológicos da moléstia de Chagas, relata sua experiência quanto à forma crônica da cardiopatia própria à doença. Dentre os aspectos analisados, este autor ressalta a freqüência das tromboses murais endocárdicas, responsáveis por fenômenos embólicos tanto na circulação pulmonar, como na sistêmica. Assim, em 126 casos de cardiopatia chagásica crônica (CCC) encontrou 160 trombos; destes, 12 se localizavam na aurícula esquerda e 66 no ventrículo esquerdo. Fenômenos embólicos secundários na circulação sistêmica foram demonstrados em 88 ocasiões, em 18 casos em vasos do encéfalo. Em relação aos 126 casos de CCC por ele analisados, estes 18 demonstram ter havido embolia em artérias encefálicas em $14,29 \%$.

O acidente vascular cerebral embólico (AVCE) como complicação da CCC foi relatado pela primeira vez na literatura por Nussenzveig \& col. ${ }^{9}$, em 1953, quando um de nós (S.-F.) avaliou do ponto de vista neurológico esse novo aspecto da doença de Chagas, até então não descrito. Ulteriormente outros autores estudaram casos mediante os quais confirmou-se essa descrição. Entre eles Sena ${ }^{11}$, Andrade ${ }^{2}$, Neiva \& Andrade ${ }^{8}$, Pereyra-Käfer \& col. ${ }^{10}$ e Badim ${ }^{4}$. Resultou demonstrar-se que o AVCE é forma comum de acometimento do sistema nervoso central (SNC) na fase crônica da moléstia de Chagas, embora secundário à $\mathrm{CCC}$ e, obviamente, não relacionado a parasitismo direto do SNC. A este último estão relacionadas outras formas da doença, cuja patogenia tem sido motivo de estudo na escola de Köberle ${ }^{\top}$.

É objeto do presente estudo relatar a freqüência em que o AVCE complicando a CCC é observado na população de pacientes que procura o Hospital das Clínicas da Faculdade de Medicina da Universidade de São Paulo (H. C.).

\section{CASUfSTICA}

A avaliação da ocorrência de AVCE dependente de CCC foi feita a partir de 100 casos consecutivos dados como de cardiopatia chagásica crônica e internados no H.C. bem como a partir de 100 casos consecutivos dados como de acidente vascular cerebral e internados na Clínica Neurológica do mesmo Hospital. Todos os casos foram levantados através do Serviço de Arquivo Médico e Estatística do H.C. (Dra. Maria Luiza de Lemos).

Trabalho da Clínica Neurológica do Hospital das Clínicas (Prof. H. M. Canelas) da Faculdade de Medicina da Universidade de São Paulo: * Docente-livre; ** Assistente. 
Da primeira amostra, o diagnóstico de CCC foi confirmado clínica e electrocardiograficamente em 76 casos. Destes, foram estudados aqueles nos quais havia positividade da reação de Machado-Guerreiro no soro e/ou em que a necrópsia confirmara a natureza chagásica da cardiopatia. Estes últimos compreendem 63 casos e deles, em cois, ocorreu AVCE $(3,17 \%)$.

Da segunda amostra, confirmou-se apresentarem acidente vascular cerebral 84 casos, de tipo não hemorrágico em 59. Foi verificada a ocorrência do AVCE na vigência de CCC em 4 pacientes, todos apresentando também positividade da reação Machado-Guerreiro no soro. Em relação ao total de pacientes com $\mathrm{AVC}$, a incidência do AVCE da CCC foi de 4,76\%; em relação aos AVC não hemorrágicos, foi de $6,78 \%$.

A análise conjunta dessas duas populações de pacientes veio a demonstrar que um deles apenas comparecia nas duas amostras: resulta o total de 5 casos, cujos dados de identificação se encontram no quadro 1 .

\begin{tabular}{cccccc}
\hline $\begin{array}{c}\text { Registro } \\
\text { (H.C.) }\end{array}$ & Sexo & Idade & Cor & $\begin{array}{c}\text { Procedencia } \\
\text { (Estado) }\end{array}$ & Condições de alta \\
\hline 635031 & $\mathrm{~m}$ & 30 & $\mathrm{~b}$ & São Paulo & Melhorado \\
909311 & $\mathrm{~m}$ & 24 & $\mathrm{pa}$ & Minas Gerais & Melhorado \\
914057 & $\mathrm{~m}$ & 65 & $\mathrm{p}$ & São Paulo & Melhorado \\
943117 & $\mathrm{P}$ & 33 & $\mathrm{~b}$ & São Paulo & Melhorado \\
951960 & $\mathrm{~m}$ & 23 & pd & São Paulo & Melhorado \\
\hline
\end{tabular}

Quadro 1 - Identificação dos pacientes: idade em anos; $m=$ masculino; $f=$ feminino; $b=$ branco; $p=$ preto; $p d=$ pardo.

\section{COMENTARIOS}

O estudo inicial sobre o assunto foi feito no mesmo Hospital e abrange 8 casos, 7 dos quais observados durante o período de dois anos ${ }^{9}$. Esses números não permitem ter idéia quanto à incidência do AVCE entre pacientes com CCC; da mesma forma, eles não permitem avaliar a incidência do AVCE próprio à CCC em relação ao total dos acidentes cerebrovasculares. A casuística apresentada nesta investigação e que compreende casos observados consecutivamente tanto de CCC como de AVC permite avaliar tais incidências em nosso meio. Dentre pacientes com cardiopatia chagásica crônica, a ocorrência de acidente vascular cerebral embólico observada foi de $3,17 \%$. Por outro lado, dentre os pacientes com acidentes cerebrovasculares, aqueles de natureza embólica secundária a cardiopatia chagásica crônica foram verificados em $4,76 \%$. E significativo o fato de entre os AVC não hemorrágicos a incidência do AVCE da CCC ter sido de 6,78\%, pois a maioria dos acidentes vasculares cerebrais embólicos, qualquer que seja sua etiopatogenia, é de tipo não hemorrágico.

A incidência ora evidenciada é inferior àquela encontrada por Andrade $^{3}$, baseada em dados de autópsia. A diferença pode decorrer de serem os fenômenos embólicos mais comuns nos estágios finais da $\mathrm{CCC}$, quando 
é manifesta a insuficiência cardíaca. Dos 18 casos desse autor, o AVC sucedeu à instalação de insuficiência cardiaca em 15. Dos 3 casos restantes, em um não havia referência a insuficiência cardíaca ou a AVC, a atrofia parietal direita encontrada podendo ter decorrido de AVCE clinicamente não manifesto. Tal fato - isto é, AVC que pela área acometida e/ou pela proporção do infarto resultante, determine sintomatologia pobre - pode ser outra razão para explicar diferenças entre casuísticas baseadas em dados anátomo-patológicos ou clínicos sobre o assunto, como as duas ora discutidas.

$E$ de notar que 4 pacientes eram do sexo masculino. Brasil ${ }^{5}$ refere que entre os pacientes com CCC por ele acompanhados, o AVCE foi mais comum em homens que em mulheres. A idade situava-se entre 23 e 33 anos em 4 pacientes. Na casuística de Andrade $^{3}$ a metade dos pacientes tinha até 40 anos de idade.

Dos 5 pacientes analisados, 4 foram hospitalizados em função da patologia cerebrovascular, isto é, dos fenômenos agudos próprios à instalação do AVCE e um em função da CCC. Brasil ${ }^{5}$ considera como fatores principais para que o coração na CCC passe a originar trombos o aumento do volume respectivo e a extrassistolia. Assim sendo, compreende-se que a gravidade do AVCE vai depender em grande parte da própria gravidade da CCC. Dessa forma pode ser explicada a diferença quanto à mortalidade observada na série anterior de casos: de 8 casos 4 faleceram ${ }^{9}$ e nesta, na qual nenhum dos pacientes faleceu.

A importância do acidente vascular embólico da cardiopatia chagásica crônica entre as manifestações da moléstia de Chagas na fase crônica, já foi apontada em diversas ocasiōes 1,6,12. Ela é salientada ainda pelos seguintes aspectos ligados à sua incidência: a ocorrência de AVCE é da ordem de $2,70 \%$ numa população de cerca de 1.000 chagásicos ${ }^{5}$; entre pacientes com CCC o AVCE ocorre em cerca de $3,17 \%$, conforme verificado neste levantamento; essa incidência aumenta para cerca de 14,29\% entre pacientes com $\mathrm{CCC}$ que vieram a falecer ${ }^{3}$; entre pacientes com acidentes cerebrovasculares o AVCE da CCC representa cerca de 4,76\% dos casos, freqüência essa que aumenta para $6,78 \%$ quando são considerados apenas os AVC não hemorrágicos, entre os quais se situa a maioria daqueles de natureza embólica.

\section{RESUMO}

A incidência de acidente vascular cerebral embólico na cardiopatia chagásica crônica foi avaliada a partir de duas séries consecutivas de casos: a primeira compreendendo 63 pacientes com cardiopatia chagásica crônica e, a segunda, 84 pacientes com acidentes cerebrovasculares, 59 dos quais de tipo não hemorrágico. Em relação aos casos de cardiopatia chagásica crônica a incidência de acidente vascular cerebral embólico mostrou-se da ordem de $3,17 \%$; em relação aos acidentes cerebrovasculares, da ordem de $4,76 \%$ e quando considerados apenas aqueles de tipo não hemorrágico, da ordem de $6,78 \%$. 


\section{Chronic Chagas' cardiopathy and cerebrovascular insults}

Mural thrombi in the cavities of the heart are commonly found in chronic Chagas' cardiopathy and embolic phenomena may result from them. Acute cerebrovascular insults resulting from embolic occlusion of cerebral arteries are described in Chagas' disease since 1953. The purpose of this investigation was to evaluate the incidence of this type of cerebrovascular embolic insult. Two groups of patients successively admitted to a general hospital were studied: the first of 63 cases of chronic Chagas' cardiopathy; the second of 84 cases of acute cerebrovascular disease. The brain insult was non-hemorrghagic type in 59 cases of the second group. The evaluation pertinent to the first series showed that embolic cerebrovascular insult occurred in $3.17 \%$ of the cases. Concerning the second series the incidence of embolic cerebrovascular insult secondary to chronic Chagas' cardiopathy was $4.76 \%$ when all cerebrovascular insults were considered, and $6.78 \%$ when only non hemorrhagic cerebrovascular insults were considered.

\section{REFERENCIAS}

1. ALENCAR, A. - Chagas' disease. In Pathology of the Nervous System, ed. por J. Minckler. McGraw-Hill Inc., Nova York, 1972, pg. 2559.

2. ANDRADE, Z, A. — Fenômenos trombo-embólicos na cardiopatia chagásica. An. Congr. Internac. Doença de Chagas (Rio de Janeiro) 1:73, 1959.

3. ANDRADE, Z. A. - Anatomia-patológica. In Doença de Chagas, ed. por J. R. Cançado. Impr. Of. Est Minas Gerais, Belo Horizonte, 1968, pg. 315.

4. BADIM, A. - Embolia cerebral em portadores de miocardite chagásica. Arq. Neuro-Psiquiat. (São Paulo) 24:28, 1966.

5. BRASIL, A. - Cardiopatia chagásica crônica. In Doença de Chagas, ed. por J. R. Cançado. Impr. Of. Est. Minas Gerais, Belo Horizonte, 1968, pg. 501.

6. FORJAZ, S. V. - Aspectos neurológicos da moléstia de Chagas: sistema nervoso central. Arq. Neuro-Psiquiat. (São Paulo) 25:175, 1967.

7. KOBERLE, F. - Aspectos neurológicos da moléstia de Chagas. Arq. Neu-ro-Psiquiat. (São Paulo) 25:159, 1967.

8. NEIVA, A. \& ANDRADE, Z. A. - Embolia cerebral em portadores de miocardite chagásica. O Hospital (Rio de Janeiro) 61:373, 1962.

9. NUSSENZVEIG, I.; SPINA-FRANÇA, A.; WAJCHEMBERG, B. L.; TIMONER, J.; MACRUZ, R. \& SERRO-AZUL, L. G. - Acidentes vasculares cerebrais embólicos na cardiopatia chagásica crônica. Arq. Neuro-Psiquiat. (São Paulo) $11: 386,1953$.

10. PEREYRA-KÄFER, J.; POCH, G. F.; MONTEVERDE, D. A.; FERNANDES BLANCO, E. \& TARSIA, O. - Neurological manifestations of the American trypanosomiasis. In Tropical Neurology, ed. por L. van Bogaert; J. Pereira-Käfer \& G. F. Poch. López Libreros Ed., Buenos Aires, 1963, pg. 237.

11. SENA, P. G. - Acidente vascular cerebral em paciente portador de cardiopatia chagásica crônica. Bol. Hosp. Clín. Fac. Med. Univ. Bahia, 2:12, 1956.

12. SPINA-F'RANÇA, A. \& MATTOSINHO-FRANÇA, L. C. - Chagas' disease and the nervous system. In Tropical Neurology, ed. por J. D. Spillane. Oxford Univ. Press, Londres, 1973, pg. 397. 